\title{
Das Paradigma des Kritischen Realismus
}

Der Kritische Realismus („Critical Realism“) ist seit Ian G. Barbours grundlegenden Issues in Science and Religion (1966) erkenntnistheoretisches Paradigma zentraler Ansätze im angelsächsischen Gespräch zwischen Theologie und Naturwissenschaften geworden; ${ }^{1}$ neben Barbour selbst sind hier insbesondere Arthur Peacocke und John Polkinghorne zu nennen. Letzterer hat sich selbst und die beiden vorgenannten unter dem Stichwort „Wissenschaftler-Theologen“ („scientist-theologians") zusammengefasst; ${ }^{2}$ alle drei Autoren haben eine erfolgreiche naturwissenschaftliche Laufbahn hinter sich gebracht, bevor sie sich der Theologie zuwandten. Als Schüler Barbours schließt sich auch der Physiker-Theologe jüngerer Generation Robert John Russell dem Kritischen Realismus an; ${ }^{3}$ der Theologe Kees van Kooten Niekerk hat eine eigene Interpretation des Kritischen Realismus zur Debatte gestellt und das Thema auch grundsätzlich behandelt. ${ }^{4}$ Er berücksichtigt dabei wie Peacocke verstärkt Vertreter eines „wissenschaftlichen Realismus“, während Barbour sich zusätzlich stark an Whitehead, Polkinghorne sich jedoch exklusiver an Michael Polanyis Philosophie orientiert. Polkinghorne bezieht sich auch auf Bernard Lonergan, dessen Philosophie an den katholischen Universitäten Nordamerikas mit diesem Stichwort verbunden wird. Eine eigene Rezeptionsschiene, nämlich den Kritischen Realismus von Roy Bhaskar, macht

1 Im angelsächsischen Bereich spricht man im Anschluss an Ian Barbours Terminologie i. d. R. vom „Science and Religion“-Diskurs, also nicht wie im deutschsprachigen Bereich von einer akademischen Diskussion zwischen zwei Disziplinen („Theologie und Naturwissenschaften“), sondern von der Debatte zwischen Wissenschaft und Religion als gesellschaftlichen Größen, wobei „Science“ als Begriff bereits naturwissenschaftlich vorbestimmt ist. Zur Diskussion, ob nun „science and religion“ oder „science and theology“ ein passenderes Etikett für den Dialog ist, beachte man den instruktiven Vortrag Michael Welkers dazu: Welker, Michael, „Science and Religion or Science and Theology“, Research Seminar given by Prof. Michael Welker v. 28.02. 2012, Faraday Institute Lectures, http://sms.cam.ac.uk/media/1219637, 23.08.2013.

2 Polkinghorne, John C., Scientists as Theologians, London: SPCK 1996.

3 Z. B. in dem Aufsatz Russell, Robert John, „Ian Barbour's Methodological Breakthrough. Creating the ,Bridge“ between Science and Theology“. In: Fifty Years in Science and Religion. Ian G. Barbour and His Legacy, hrsg. v. dems., Aldershot Hants: Ashgate 2004, 45-60.

4 van Kooten Niekerk, Kees, „A Critical Realist Perspective“. In: Rethinking Theology and Science. Six Models for the current Dialogue, hrsg. v. N. H. Gregersen/J. W. van Huyssteen, Grand Rapids: Eerdmans 1998, 51-86; ders., „Critical Realism“. In: Encyclopedia of Science and Religion, hrsg. v. J. W. van Huyssteen, Bd. I, New York: Macmillan 2003, 190 -193; Ders., „Critical Realism in Theology and Science“. In: Encyclopedia of Sciences and Religions, hrsg. v. A. Runehov/L. Oviedo/N. Azari, Dordrecht: Springer 2013, 554-558. 
der Biochemiker und Theologe Alister McGrath für das Gespräch von Theologie und Naturwissenschaften fruchtbar. ${ }^{5}$ Auch der finnische Philosoph Ilkka Niiniluoto vertritt einen kritischen wissenschaftlichen Realismus („critical scientific realism“), doch hat dieser keinen Bezug zum Theologie-NaturwissenschaftenDialog. 6

Im deutschsprachigen Gespräch der Disziplinen wird ein kritischer Realismus (im Anschluss an Polkinghorne) von dem ev. Theologen Michael Welker vertreten, ${ }^{7}$ es gibt aber auch wichtige Positionen, die einen Nominalismus (Hübner ${ }^{8}$, Link ${ }^{9}$ ) oder Konstruktivismus (Audretsch ${ }^{10}$, Körtner ${ }^{11}$ ) propagieren oder diesen Positionen nahestehen. So ist die erkenntnistheoretische Front hier so zersplittert wie in der Philosophie selbst, auch wenn kritische Realisten gerne behaupten, dass ihre eigene Position in der Philosophie fast aller Zeiten vorherrschend sei. ${ }^{12}$ Von Barbour und Polkinghorne wird ähnlich angenommen, der (kritische) Realismus sei Mehrheitsposition unter praktizierenden Wissenschaftlern; ${ }^{13}$ tatsächlich legen

5 McGrath, Alister E., A Scientific Theology, Bd. II: Reality, Grand Rapids: Eerdmans 2002.

6 Niiniluoto, Ilkka, Critical Scientific Realism, Oxford: Oxford University Press 1999.

7 Polkinghorne, John C./Welker, Michael, An den lebendigen Gott glauben, Gütersloh: Gütersloher Verlagshaus 2005, $181 \mathrm{ff}$. Auch Hans-Peter Grosshans vertritt einen theologischen Realismus. Er rückt die Bedeutung einer realistischen Sprachtheorie in den Mittelpunkt seiner Untersuchung, verweist auf die Rezeptionsschiene Barbours aber nur in einer Anmerkung: Grosshans, Hans-Peter, Theologischer Realismus, Tübingen: Mohr Siebeck 1996, 5 Anm. 10.

8 Hübner, Jürgen, Theologie und biologische Entwicklungslehre. Ein Beitrag zum Gespräch zwischen Theologie und Naturwissenschaft, München: C. H. Beck 1966; ders., „Die Wirklichkeit der Natur im Streit zwischen Religion und Wissenschaft“. In: Urknall oder Schöpfung? Zum Dialog von Naturwissenschaften und Theologie, hrsg. v. W. Gräb, Gütersloh: Gütersloher Verlagshaus 1995, 89-100.

9 Link, Christian, „Die Wahrnehmung der Natur als Schöpfung. Zum Modell eines Dialogs zwischen Naturwissenschaft und Theologie im Anschluss an Viktor von Weizsäcker“. In: Zufall Mensch? Das Bild des Menschen im Spannungsfeld von Evolution und Schöpfung, hrsg. v. L. Klinnert, Darmstadt: WBG 2007, 55-71.

10 Audretsch, Jürgen, Die sonderbare Welt der Quanten, München: C. H. Beck 2008.

11 Körtner, Ulrich J., ,,Schöpfung“ und ,Evolution‘: Quasi dasselbe mit anderen Worten? Zur Hermeneutik des Dialogs zwischen Theologie und Naturwissenschaften“. In: Glaube und Denken 23 (2010), 11-38, bes. 26.

12 So z. B. Külpe, Oswald, Einleitung in die Philosophie, Leipzig: Hirzel ${ }^{7} 1915,163$.

13 „Almost all scientists believe that they are learning about the actual nature of the physical world that they investigate. Consciously or unconsciously, they are critical realists" (Polkinghorne, John C., „The Metaphysics of Divine Action“. In: Chaos and Complexity, hrsg. v. R. J. Russell/A. Peacocke/N. Murphy, Notre Dame: University of Notre Dame Press 1995, 147-156, hier 148); „Scientists usually assume realism in their work“ (Barbour, Ian G., Issues in Science and Religion, London: SCM 1966, 171); „Most scientists are incurably realists“ (ders., Religion and Science. 
eine Umfrage ${ }^{14}$ und die Wissenschaftsgeschichte ${ }^{15}$ eine solche Annahme nahe, doch ist ein Mehrheitsvotum sicher kein Garant für die Wahrheit des Ansatzes.

„Kritischer Realismus“ ist ebenso wenig wie „critical realism“ ein eindeutiger Begriff. Eine ganze Vielzahl von einander verwandter, vom Ursprung her aber verschiedener philosophischer Konzeptionen gebraucht diesen Begriff, und noch mehr Positionen werden gelegentlich so bezeichnet, ohne dass diese den Begriff überhaupt selbst benutzen. Eine Erklärung für dieses Phänomen könnte darin liegen, dass es sehr naheliegend ist, sich von einem angenommenen naiven Realismus durch das Attribut „kritisch“ zu distanzieren und damit gleichzeitig eine vorhandene realistisch orientierte Position $\mathrm{zu}$ qualifizieren. ${ }^{16}$ Man könnte allerdings fragen, ob dieses Vorgehen schon der philosophischen Reflexion letzter Schluss sein kann.

\section{Was behauptet der kritische Realismus?}

Allen hier vorgestellten kritisch-realistischen Positionen gemeinsam ist 1.) ein neues Verständnis von „Realismus“ im Sinne der kantischen Vernunftkritik. Kant ist es, der „die zuvor in Hinsicht auf den Seinsstatus und die Gegebenheitsweise der Universalien gebrauchten Ausdrücke ,Realist“ und ,Realismus““ nun „auf die Frage nach der Existenz und der Existenzweise der raumzeitlichen Welt und nach dem Verhältnis von Erscheinung und Ansichsein“ bezieht. ${ }^{17}$ Kant selbst vertritt bekanntlich einen transzendentalen Idealismus, damit ist allerdings auch ein

Historical and Contemporary Issues. A Revised and Expanded Edition of Religion in an Age of Science, San Francisco: Harper Collins 1997, 118).

14 Eine Ad-hoc-Umfrage unter interessierten Lesern des Journals Physics World (April 2002) führte zu dem Ergebnis, dass viele (wenn nicht die meisten) Leser die Grundannahmen des von Polkinghorne postulierten kritischen Realismus in der Tat teilen würden.

15 Vgl. dazu McGrath, Reality (2002), 142: „Yet historically, instrumentalist understandings of science have often transmuted into realist understandings with the passing of time."

16 Daher sind auch noch mehr Verwendungen des Begriffs zu finden, als in diesem Aufsatz aufgeführt werden, so z. B. McCormack, Bruce, Karl Barth's Critically Realistic Dialectical Theology. Its Genesis and Development 1909-1936, Oxford: Clarendon 1995; Padgett, Alan G., Science and the Study of God. A Mutuality Model for Theology and Science, Grand Rapids: Eerdmans 2003; Wright, Nicholas Thomas, The New Testament and the People of God, London: SPCK 1992.

17 Halbfass, Wilhelm, Art. „Realismus II. - 1. Realismus vs. Idealismus“. In: Historisches Wörterbuch der Philosophie, hrsg. v. J. Ritter/K. Gründer, Bd. VIII, Basel: Schwabe 1992, Sp. 156 159, hier Sp. 156. 
empirischer Realismus verbunden: ${ }^{18}$ „Das Ding an sich besteht zwar, ist aber nicht erkennbar““ ${ }^{19}$ Kant kritisiert die Position des „transzendentalen Realismus“, ${ }^{20}$ die in etwa der des kritischen Realismus entspricht und die einen zwar eingeschränkten, jedoch möglichen Zugriff auf das Ding an sich postuliert. ${ }^{21}$ Von einem kritischen Realismus kann man daher überall dort reden, wo eine Art Synthese von Epistemologie und Ontologie angestrebt wird. ${ }^{22}$ Gegenteil dieser Art des Realismus ist nicht wie in der mittelalterlichen Diskussion der Nominalismus, sondern der Idealismus, oder was als dessen moderne Entsprechung angesehen wird. Nicht nur im katholischen Bereich wird jedoch auch eine Kontinuität der Realismen gedacht, so dass auch der Nominalismus als Gegenpart auftaucht. Damit wird 2.) eine weitere Gemeinsamkeit aller kritisch-realistischen Positionen deutlich, die in der Einnahme einer Mittelposition zwischen den Extremen, dem „naiven Realismus“ auf der einen und dem Idealismus oder modernen Konstruktivismus auf der anderen Seite, besteht. Außerdem besteht 3.) eine Gemeinsamkeit der meisten hier untersuchten kritischen Realismen in der Idee einer Schichtenordnung der Wirklichkeit, die nichtreduktionistisch interpretiert wird. Ob dies ein intrinsisches oder zufälliges Attribut aller kritischen Realismen ist, wäre eine eigene ideengeschichtliche Untersuchung wert. ${ }^{23}$

18 Kant, Immanuel, Kritik der reinen Vernunft. Nach der ersten und zweiten Originalausgabe hrsg. v. J. Timmermann, Hamburg: Meiner 1998, 486 [A371]. Im Folgenden zitiert als: Kant, KrV. 19 Eisler, Rudolf, „Realismus“. In: Ders., Wörterbuch der philosophischen Begriffe, Bd. II, 4. Aufl., Berlin: Mittler 1929, 622-625, hier 623.

20 Kant, KrV, $484 \mathrm{ff}$. [A369ff.].

21 Aloys Wenzl sieht dennoch einen Unterschied zwischen transzendentalem und kritischem Realismus, nämlich in der Rolle, die Kant dem Raum, der Zeit und den Kategorien zuweist. „Was Kant hier dem ,transzendentalen Realisten' unterstellt ist jedenfalls nicht die Lehre des kritischen Realisten, der Raum und Zeit ja ebenfalls nicht als Gegenstände an sich betrachtet, aber freilich auch nicht nur als Formen unserer Anschauung, sondern als Erscheinungen eines ihnen zugrunde liegenden realen Beziehungsgefüges, dem die wahrgenommenen raumzeitlichen Beziehungen entsprechen“ (Wenzl, Aloys, „Der kritische Realismus als erkenntnistheoretische Synthese und als Aufgabe der Gegenwart“. In: Forschungen und Fortschritte 28/4 (1954), 97-99, hier 97).

22 Polkinghornes Formel des kritischen Realismus „epistemology models ontology“ hat also ihre Berechtigung, worauf ich noch eingehen werde. Der Bezug zu Kant mag den Polkinghornekenner verwirren, weil sich dieser eher von Kant distanziert (Polkinghorne, John C., An Gott glauben im Zeitalter der Naturwissenschaften, Gütersloh: Gütersloher Verlagshaus 2000, 57), doch ist wie dargestellt der Bezug des kritischen Realismus auf die „Frage nach der Existenz und der Existenzweise der raumzeitlichen Welt“ ein Erbe von eben dessen Philosophie.

23 Vgl. einige Untersuchungen in Losch, Andreas, Jenseits der Konflikte, Göttingen: Vandenhoeck \& Ruprecht 2011, $129 \mathrm{f}$. 
Ferner wäre zu fragen, inwiefern nicht 4.) alle kritischen Realismen die Möglichkeit eines erkenntnistheoretischen Fortschritts unterstellen, und damit dem Konzept der zunehmenden Wahrheitsähnlichkeit zumindest zugeneigt sind. Wie anders soll man das Postulat der Überwindung eines „naiven“ durch den „kritischen“ Realismus erkenntnistheoretisch verstehen?

\subsection{Welcher kritische Realismus ist gemeint?}

Wenn vom kritischen Realismus die Rede ist, muss nach Feststellung der grundsätzlichen Ähnlichkeiten als nächstes gefragt werden: Welcher kritische Realismus ist gemeint? Die Tatsache, dass der Begriff immer wieder neu erfunden worden ist, verrät viel über die Rezeptionsreichweite der jeweiligen Konzeptionen des Begriffs, denn bei Kenntnis einer bereits so bezeichneten Position wird man den eigenen Neuansatz sicher nicht ebenfalls so bezeichnen bzw. ihn wenigstens in Beziehung zu dem bereits so Bezeichneten setzen. Ich will daher zunächst einen groben Überblick über die existierenden kritischen Realismen geben, bevor ich mich auf den kritischen Realismus im Gespräch von Theologie und Naturwissenschaften konzentriere. Aufgrund der großen Menge als kritisch realistisch klassifizierter Positionen beschränke ich mich bei dem Überblick auf diejenigen Positionen, die diesen Begriff auch selbst benutzen.

Im engeren Sinne bezieht sich der „Kritische Realismus“ in der 1.) deutschen Philosophie auf eine Position, die u. a. von C. Stumpf, O. Külpe, E. Becher und A. Messer vertreten wurde. Charakteristisch für diese Schule, die auch von Wilhelm Wundt beeinflusst worden ist, sind die Unterscheidung zwischen naivem und kritischem Realismus und die Überzeugung, die vorherrschende Position in der Philosophie fast aller Zeiten darzustellen. ${ }^{24}$ Das wäre allerdings auch anhand der deutschen Geistesgeschichte zu überprüfen und aufgrund des fortdauernden Einflusses Kants sicher nicht unzweifelhaft.

Im 2.) angelsächsischen Bereich gibt es eigene Prägungen des Begriffs. So einmal a) der „critical realism“ des britischen Philosophen Dawes Hicks, ${ }^{25}$ daneben b) der „critical realism“ Roy Wood Sellars’ und seiner Weggefährten, die damit versuchten, die Einsichten der vorangegangenen Bewegung des „New Realism“

24 Vgl. Anm. 12.

25 Nach eigener Auskunft hat Hicks den Begriff bereits „lange vor“ den amerikanischen kritischen Realisten benutzt (Hicks, G. Dawes, Critical Realism. Studies in the Philosophy of Mind and Nature, London: Macmillan 1938, v). Sein erstes Essay zu dem Thema sei nämlich bereits 1917 veröffentlicht worden. Allerdings hat Roy Wood Sellars bereits 1908 von dem Begriff Gebrauch gemacht (vgl. Losch, Jenseits der Konflikte (2011), 111 Anm. 42). 
mit denen des Idealismus zu vereinbaren. Die genannten Autoren hatten jedoch keinen direkten Einfluss auf die Entwicklung des kritischen Realismus im Gespräch von Theologie und Naturwissenschaften. Roy Wood Sellars' Sohn Wilfrid Sellars, ein Vertreter des wissenschaftlichen Realismus, wird allerdings von Arthur Peacocke mit einem Artikel zitiert, der die Familientradition des Vaters weiterführt, ${ }^{26}$ von daher kann man von einem indirekten Einfluss reden. Schließlich ist insbesondere auf nordamerikanischem katholischem Gebiet - der kritische Realismus c) Bernard Lonergans zu nennen, ${ }^{27}$ der im Gespräch von Theologie und Naturwissenschaften vom Anglikaner John Polkinghorne rezipiert worden ist.

Der 3.) finnische Philosoph Ilkka Niiniluoto vertritt einen „critical scientfic realism“ und kürzt diesen manchmal auch zu „critical realism“ ab. Man kann ihn am besten als exponierten wissenschaftlichen Realisten charakterisieren, dessen positivistisch orientierter Ansatz ${ }^{28}$ allerdings bislang keinerlei Einfluss auf das Gespräch von Theologie und Naturwissenschaften gehabt hat. Eine relativ neue Verwendung erfährt der Begriff des kritischen Realismus als 4.) Bezeichnung der Philosophie Roy Bhaskars. Der Autor selbst verweist darauf, dass er seine allgemeine Wissenschaftsphilosophie „transzendentaler Realismus“ und seine spezielle Philosophie der Geisteswissenschaften „kritischer Naturalismus“ genannt hatte. „Nach und nach begannen die Leute, die zwei zu vermengen und auf den Hybrid als ,Kritischen Realismus‘ zu verweisen.“" ${ }^{29}$ Derjenige, der Roy Bhaskars Philosophie für das Gespräch zwischen Theologie und Naturwissenschaften fruchtbar gemacht hat, ist Alister McGrath in seiner Scientific Theology, wobei es allerdings wenig Sinn macht, den früheren Gesprächspartnern mit McGrath die Nichtbeachtung Bhaskars vorzuwerfen, ${ }^{30}$ weil die Prägung des Begriffs durch Ian Barbour, wie darzustellen ist, wesentlich früher erfolgte.

26 Sellars, Wilfrid, „Phenomenalism“. In: Ders., Science, Perception and Reality, London: Ridgeview 1963, 60 -105. Vgl. dazu Losch, Jenseits der Konflikte (2011), 118.

27 Lonergan, Bernhard J. F., Method in Theology, London: Darton, Longman \& Todd 1972; ders., Critical Realism and the Integration of the Sciences. Six Lectures at University College. Unveröffentlichtes Manuskript, Dublin 1961; zur Auseinandersetzung Lonergans mit dem thomistischen Realismus Gilsons siehe Armour, Paul St., „Lonergan and Gilson on the Problem of Critical Realism“. In: The Thomist 69 (2005), 557-592.

28 So das Urteil von Ernan McMullin, vgl. McMullin, Ernan, „Review of ,Is Science Progressive?““. In: Isis 78 (1987), 260-261.

29 Bhaskar, Roy, Reclaiming Reality, London: Routledge 1989, 190: „Gradually people started to elide the two und refer to the hybrid as ,critical realism."“

30 Vgl. McGrath, Reality (2002), $207 \mathrm{f}$. 


\subsection{Barbours Begriffsprägung}

Ian Barbour, der den Begriff „critical realism“ in die Debatte von Theologie und Naturwissenschaften eingeführt hat, waren keine der vorgenannten Positionen bekannt. Er hatte bereits früh darüber nachgedacht, dass sowohl der Wissenschaftler wie der Betende einen Realismus annehmen, also von der Wirklichkeit ihres Gegenstands bzw. Gegenübers ausgehen, ${ }^{31}$ und postulierte im Gespräch mit F. S. C. Northrop in der Erstausgabe von Zygon sodann eine Art „kritischen Realismus“, der besage:

„Ja, Wissenschaft versucht die Wirklichkeit zu beschreiben, doch sie tut dies nur sehr indirekt in höchst symbolischer und abstrakter Sprache.“ Man muss Modelle benutzen, doch man muss ihre Grenzen berücksichtigen; man muss begreifen, dass sie partiell und begrenzt sind, dass jedes bestimmte Aspekte auswählt und diese betont, so dass keines von ihnen in einfacher Weise mit der Wirklichkeit übereinstimmt. ${ }^{32}$

In seinen Issues in Science and Religion von 1966 (was die Modelle angeht, ausführlicher in dem späteren Myth, Models and Paradigms ${ }^{33}$ ) führt er diese Idee dann aus und vertritt nun diesen kritischen Realismus, offenbar ohne dass ihm die vorige Sellars'sche Begriffsbenutzung bekannt ist. ${ }^{34}$ Stattdessen bezieht er sich auf die aufkommende Bewegung des wissenschaftlichen Realismus und attribuiert seine Idee eines kritischen Realismus darüber hinaus der realistisch orientierten Philosophie Whiteheads.

Grundidee des kritischen Realismus im Ansatz Barbours ist diejenige einer Mittelposition $^{35}$, die nach seinen Worten Positivismus, Instrumentalismus und Idealismus gleichermaßen vermeidet bzw. eine Synthese von naivem Realismus

31 Barbour, Ian G., „The Methods of Science and Religion“. In: Science Ponders Religion, hrsg. v. H. Shapley, New York: Appleton-Century-Crofts 1960, 196-215, hier 203.

32 Barbour, Ian G., „Commentary on Theological Resources from the Physical Sciences“. In: Zygon 1/1 (1960), 27-30, hier 30: „Avoiding these extremes, I would tend to end up where Northrop does with a kind of critical realism that says, ,Yes, science is trying to describe reality, but it does so only very indirectly in highly symbolic and abstractive language.' One has to use models, but one has to recognize their limitations; one has to realize that they are partial and limited, that each one selects certain aspects and emphasizes those that none of them corresponds exactly in any simple way to reality."

33 Barbour, Ian G., Myths, Models and Paradigms. A Comparative Study in Science and Religion, New York: Harper Collins 1974.

34 "I had run across Wilfrid Sellars work in philosophy of science but not Roy Wood Sellars' broader epistemology“ (Barbours E-Mail an den Verfasser vom 8. März 2008).

35 Vgl. dazu auch Losch, Jenseits der Konflikte (2011), Kap. 3. 
und Instrumentalismus darstellt. ${ }^{36}$ Er definiert den kritischen Realismus so, dass er

„beides anerkennen muss, die Kreativität des menschlichen Geistes und die Existenz von Mustern in Ereignissen, die nicht durch den menschlichen Geist erschaffen sind. Ein Kritischer Realismus erkennt die Indirektheit der Referenz und die realistische Intention der Sprache, wie sie in der scientific community benutzt wird, an“ (IB/172). ${ }^{37}$

Eine weitere Beschreibung charakterisiert den kritischen Realismus als solchen, in dem sowohl Subjekt wie Objekt im Erkenntnisprozess eine Rolle eingeräumt wird (deswegen „kritisch“), die Priorität jedoch auf dem Beitrag des Objektes liegt (deswegen „Realismus“) (IB/171). Barbour begründet dies damit, dass „unser primitives Bewusstsein“ dasjenige sei, „in einer Welt zu sein, nicht sie zu konstruieren“ (IB/171). Auf einer tieferen Ebene - und hier kommt Barbours Whiteheadrezeption ins Spiel - beschreibt der kritische Realismus nach Barbour nämlich ein „Bewusstsein unserer selbst, das aus einer Übereinstimmung, Verbindung und Teilhabe an Prozessen entsteht, die uns übersteigen“ (IB/171). ${ }^{38}$

\subsection{Parallelität von Wissenschaft und Religion}

Von Whitehead inspiriert ist Barbours Idee der gleichzeitigen Anwendung dieses erkenntnistheoretischen Paradigmas auf das Gebiet der Theologie. Zentrales und von Barbour wiederholt in Anspruch genommenes Zitat ist hier eine Passage aus Religion in the Making, die eine epistemologische Parallelität der beiden Domänen suggeriert:

Die Dogmen der Religion sind Ansätze, die in der religiösen Erfahrung der Menschheit enthüllten Wahrheiten präzise zu formulieren. Auf genau dieselbe Weise sind die Dogmen

36 Barbour, Ian G., „Theology and Physics Forty Years Later“. In: Zygon 40/2 (2005), 507-511, hier 508; Barbour, Issues (1966), 206. Im Folgenden zitiert durch IB/Seitenangaben in Klammern. 37 Orig.: „must acknowledge both the creativity of man's mind, and the existence of patterns in events that are not created by man's mind. Critical realism acknowledges the indirectness of reference and the realistic intent of language as used in the scientific community“.

38 Orig.: „consciousness of ourselves as arising out of rapport, interconnection and participation in processes reaching beyond ourselves“. Zur Verwendung dieses Zitates vgl. Losch, Jenseits der Konflikte (2011), Kap. 5.6. Es stammt ursprünglich von Emmet, Dorothy, The Nature of Metaphysical Thinking, London: Macmillan 1945, 65. 
der Physik Versuche, die in der Sinneswahrnehmung der Menschheit freigelegten Wahrheiten präzise zu formulieren (IB/129, 208). ${ }^{39}$

In diesem Sinne sieht Barbour dann auch als eine Gemeinsamkeit der wissenschaftlichen wie der religiösen Gemeinschaft an, dass die interpretative Sprache in beiden Gemeinschaften „,realistisch und referentiell benutzt“ (IB/267) werde, ${ }^{40}$ ein Echo seiner eingangs beschriebenen Anfangsidee. Er erkennt vier Parallelen zwischen Wissenschaft und Religion: 1.) Die Tatsache, dass es in beiden Fällen keine uninterpretierte Erfahrung gibt, weiterhin 2.) die Bedeutung der Gemeinschaft und ihrer Paradigmen, ${ }^{41}$ 3.) die Verwendung von Modellen und Analogien in Wissenschaft wie Religion und schließlich 4.) die Tatsache, dass in beiden Bereichen verbundene Netzwerke von Konzepten gemeinsam bewertet und die Kriterien der Stimmigkeit, des Umfangs und der Angemessenheit gegenüber der Erfahrung benutzt werden (IB/210, 267). ${ }^{42}$

Ebenso erkennt Barbour natürlich auch einige Unterschiede zwischen den Gebieten an. Er gesteht zu, 1.) dass der Grad der persönlichen Beteiligung in der Religion größer als in der Wissenschaft ist. Auch die 2.) historische Offenbarung habe keine Parallele in der Wissenschaft, ebenso wie die 3.) nichtkognitiven Funktionen religiöser Modelle. Schließlich sei die 4.) intersubjektive Überprüfbarkeit von religiösen Anschauungen verglichen mit wissenschaftlichen Theorien/Paradigmen stark eingeschränkt (IB/267). ${ }^{43}$

Grundsätzlich festzuhalten bleibt aber die in diesem Modell suggerierte und von Whitehead (und auch Michael Polanyi) inspirierte Spektrumsthese, dass Wissenschaft und Religion auf ein und demselben Spektrum zu finden sind, nur der Anteil des Subjektiven und Objektiven am Erkenntnisprozess jeweils differiere, wobei die Subjektivität in Richtung Religion zunehme. Die Geisteswissenschaften werden auf diesem Spektrum dann zwischen Naturwissenschaft und Religion verortet, aber nicht qualitativ abgesondert:

39 Barbour zitiert hier Whitehead, Alfred North, Wie entsteht Religion?, Frankfurt a. M.: Suhrkamp 1990; englische Fassung: Whitehead, Alfred North, Religion in the Making, New York: Meridian Books 1960, 57.

40 Dies erinnert an Barbours ursprüngliche Idee, vgl. den Anfang des Absatzes „Barbours Begriffsprägung“ in diesem Text.

41 Siehe dazu Barbour, Myths, Models and Paradigms (1974).

42 Für eine detailliertere Aufstellung der Parallelen siehe Losch, Jenseits der Konflikte (2011), $139-141$.

43 Siehe auch Losch, Jenseits der Konflikte (2011), 141. 
Wir finden keine scharfe Linie, welche die Unterscheidung der akademischen Welt in zwei entgegengesetzte Lager rechtfertigen würde, sondern eher ein „Spektrum“, in dem die Rolle des subjektiven Lebens des Menschen stark variiert (IB/188). ${ }^{44}$

Barbour sieht sich durch die zunehmende Bedeutung der Kategorie des Geschichtlichen auch in den Naturwissenschaften später in seinen die Unterschiede nivellierenden Annahmen bestätigt. ${ }^{45}$ Die anerkannten Kriterien von Wissenschaftlichkeit lassen sich seiner Ansicht nach alle auf die Religion anwenden; dies sind die Kriterien 1.) der Übereinstimmung mit den Daten, 2.) der Stimmigkeit, 3.) des Umfangs und 4.) der Fruchtbarkeit (IB/144ff.). ${ }^{46}$ Wie Barbour diese im Einzelnen - zwar gebrochen, aber grundsätzlich auch - in der Religion erfüllt sieht, soll hier nun nicht ausgeführt werden; es bleibt aber festzuhalten, dass damit die Grundidee der Parallelität der Bereiche, wie sie Whitehead vorgedacht hat, zum Ausdruck gebracht wird. Credo des Kritischen Realismus in der Ausgestaltung Barbours ist dies: Obwohl die Gegensätze zwischen Wissenschaft und Religion immer in Erinnerung behalten werden müssen, sind diese Gegensätze nicht so absolut wie die meisten gegenwärtigen Theologen und Philosophen behauptet haben (IB/268). Der kritische Realismus Barbours ist ein Theorieansatz, der versucht, ein zur Zeit der Publikation der Issues auch im angelsächsischen (wissenschaftlichen) Bereich vorherrschendes Modell der Unabhängigkeit von Religion und Wissenschaft zu überwinden. Spuren davon sind in den Issues dort zu finden, wo Barbour den für dieses Modell typischen Zwei-Sprachen-Ansatz als Ausgangspunkt wählt, um in der Erforschung verschiedener Gegenstandsbereiche dann aber weiter fortzuschreiten und diesen hinter sich zu lassen (IB/268f.).

\subsection{Peacockes Aufnahme des Begriffs}

Arthur Peacockes Gebrauch des kritischen Realismus liegt auf einer Linie mit Ian Barbour, auch wenn er Putnam ${ }^{47}$ und andere wissenschaftliche Realisten ${ }^{48}$ als Gewährsleute heranzieht. Der naive Realismus wird bei Peacocke weniger als philosophische denn als zeitgenössische Haltung dargestellt:

44 Orig.: „We find no [...] sharp line that would justify the division of academia into two opposing camps, but rather a ,spectrum ' within which the role of man's subjective life varies greatly."

45 Barbour, Ian G, Religion and Science. Historical and Contemporary Issues. A Revised and Expanded Edition of Religion in an Age of Science, San Francisco: Harper 1997, 137.

46 Ebd., 113, 158. Eine frühe Version der Kriterien erscheint in Barbour, Issues (1966), wo Umfang und Fruchtbarkeit anscheinend unter der Überschrift „comprehensiveness“ vereint sind. 47 Peacocke, Arthur, Creation and the World of Science, Oxford: Clarendon 1979, 21.

48 Ders., Intimations of Reality, Notre Dame: University of Notre Dame Press 1984, 21-29. 
Die Perspektive der meisten Laien und vieler Wissenschaftler auf wissenschaftliche Konzepte und Mechanismen ist, dass es sich dabei um wörtliche Beschreibungen der Welt handelt, Reproduktionen der objektiven Wirklichkeit, hauptsächlich basierend auf der Annahme, dass „Wissenschaft funktioniert“.49

Peacocke kontrastiert diese Ansicht mit den Entwicklungen in der Wissenschaftsphilosophie, dem „Standardansatz“ der Struktur wissenschaftlicher Theorien bis in die 1970er, den danach aufkommenden sozio-kontextuellen Ansätzen wissenschaftlicher Theorien (beeinflusst von Thomas Kuhns Structure of Scientific Revolutions) und der anschließenden Aufteilung in Befürworter einer Soziologie wissenschaftlichen Wissens und Anhänger des wissenschaftlichen Realismus. Der große Vorteil des wissenschaftlichen Realismus nach Peacocke ist es, dass sich die Wissenschaftsphilosophie hier damit beschäftigt hat, wie Wissenschaft tatsächlich praktiziert wird.

Er postuliert zunächst einen „skeptischen und qualifizierten“ Realismus, ${ }^{50}$ übernimmt dann aber auch den von Barbour geprägten Begriff des „kritischen Realismus“. Nach Peacocke bedeutet ein solcher Realismus, dass

„Modelle und Hypothesen als ,Kandidaten für Wirklichkeit‘ betrachtet werden, das bedeutet,
als Modelle und Hypothesen einer wirklichen (aber unvollkommen gewussten) Welt, der sich
die Modelle annähern und auf die die Hypothesen verweisen“. ${ }^{.5}$

Peacocke ist damit insbesondere von der Darstellung der Bedeutung von Modellen, wie sie Barbour in Myths, Models and Paradigms entwickelt hat, abhängig. ${ }^{52}$ Von diesem übernimmt er auch die Idee, dass ein solcher Realismus in beiden Bereichen, Wissenschaft und Religion, Anwendung finden kann, und dazu zählt auch der Aspekt, dass ihre Modelle „die Wirklichkeit widerspiegeln und ernst, aber nicht wörtlich genommen werden wollen“ ${ }^{53}$ Eine weitere Ähnlichkeit, die er aufführt, ist diejenige, dass Modelle in beiden Bereichen weniger damit beschäftigt sind, Gegenstände abzubilden, als Muster von Beziehungen darzu-

49 Ebd., 15: „... most laymen's, and many scientists', view of scientific concepts and mechanisms is that they are literal descriptions of the world, reproductions of objective reality, mainly on the basis that ,science works'.“

50 Peacocke, Creation (1979), 21.

51 Ebd.: „Models und hypotheses are regarded as ,candidates for reality', that is, models of, hypotheses about, a real but only imperfectly known world to which the models approximate and the hypothesis genuinely refer.“

52 Vgl. Losch, Jenseits der Konflikte (2011), $142 \mathrm{f}$.

53 Peacocke, Intimations (1984), 42: „They reflect reality and are to be taken seriously but not literally.“ Bereits Barbour benutzt den Ausdruck „Ernst, aber nicht wörtlich“, vgl. dazu Losch, Jenseits der Konflikte (2011), 114 Anm. 61. 
stellen. Wie Barbour betont er schließlich die Rolle der Gemeinschaft als „lebendige Referenztradition“ für Modelle in beiden Bereichen. ${ }^{54}$

Peacocke kennzeichnet - ebenfalls ähnlich wie Barbour - auch einige Unterschiede zwischen Wissenschaft und Religion. Die biblische Wurzelmetapher ${ }^{55}$ Gottes als personaler Quelle allen Seins habe eine umfassende Rolle an der Spitze einer Hierarchie theologischer Modelle und Metaphern, die religiöse Erfahrung erläutern, und in der Wissenschaft nehme keine wissenschaftliche Theorie diese Rolle wahr. Daher stimmt er mit Barbour darin überein, dass religiöse Modelle und ihre assoziierten Metaphern einflussreichen und abstrakten Theorien (Doktrinen) weniger untergeordnet sind als Modelle in Verhältnis zu wissenschaftlichen Theorien. Ein zweiter Unterschied, den Peacocke anmerkt, ist der, dass religiöse Modelle eine stark affektive Funktion haben, die eine moralische und spirituelle Antwort herausfordert und die Verpflichtung und Selbstbeteiligung hervorruft. Dennoch möchte Peacocke - wieder wie Barbour - nicht die kognitive Rolle von theologischen Modellen herunterspielen: „Christliche Gläubige nehmen von ihren Modellen an, dass sie die Wirklichkeit abbilden, sonst wären sie affektiv und persönlich ineffektiv und unwirksam“.56 Hier wird Peacockes zentraler Punkt deutlich, welcher in der referentiellen Funktion von Modellen in sowohl Wissenschaft als auch Religion besteht, wie er für jede realistische Position wesentlich ist.

\subsection{Polkinghornes Paradigma}

Bereits in seinem frühen und grundlegenden Werk One World vertritt Polkinghorne eine Perspektive auf die Wissenschaft, die deren Errungenschaften zugesteht, ein „zunehmender Zugriff auf eine tatsächliche Wirklichkeit“ zu sein. ${ }^{57}$ Dies ist eindeutig eine Option für einen (konvergenten) Realismus. Da nun Polkinghornes Ansicht nach alles, was erreicht werden kann, Wahrheitsähnlichkeit (,versimilitude“), nicht Wahrheit, ist, definiert Polkinghorne diesen Realismus als einen kritischen. Er macht dabei allerdings nicht deutlich, wo er den Begriff

54 Peacocke, Intimations (1984), 42.

55 Dieses Konzept übernimmt Peacocke von McFague, Sallie, Metaphorical Theology. Models of God in Religious Language, Philadelphia: Augsburg Fortress 1982, 104.

56 Peacocke, Intimations (1984), 44: „Christian believers take their models to depict reality, otherwise they would be affectively and personally ineffectual and inoperative."

57 Polkinghorne, John C., One World. The Interaction of Science and Theology, London: SPCK 1986, 22, 24: „tightening grasp of an actual reality“. 
hernimmt, ${ }^{58}$ so dass sich nur vermuten lässt, dass er diesen von Ian Barbour übernommen hat, dessen Issues er in One World als eine seiner Hauptquellen angibt. Später weitet Polkinghorne seine Aufmerksamkeit auch auf Peacockes Intimations of Reality und Bernard Lonergans Werk, das den Begriff ebenfalls benutzt, aus. Eine beliebte Metapher Polkinghornes im Zusammenhang mit dem kritischen Realismus ist diejenige der Landkarte. Ziel der Wissenschaft ist demnach „eine angemessene Darstellung eines umschriebenen physikalischen Bereichs, eine Karte, die gut genug für einige, aber nicht für alle Zwecke ist“ “. ${ }^{59}$ Als für die Beschreibung relevant erscheint bei Polkinghornes Wissenschaftsverständnis jedoch nicht die Ebene der Modelle, sondern diejenige von Theorien. Modelle sind „wertvoll, aber von bekannter Inadäquatheit“, ${ }^{60}$ während Theorien die Kandidaten für eine wahrheitsähnliche Beschreibung der physischen Wirklichkeit sind. ${ }^{61}$

Polkinghorne ist auf eigenen Wegen zu einer recht interessanten Beschreibung des kritischen Realismus gelangt; er fasst diesen mit dem Motto zusammen, dass demnach die Epistemologie die Ontologie modelliert bzw. abbildet: ${ }^{62}$ die Gesamtheit dessen, was wir wissen können, ist ein verlässlicher Führer zu dem, was der Fall ist. ${ }^{63}$ Polkinghornes Paradigma für dieses Motto ist sein Verständnis der Unschärferelation. Ursprünglich war es epistemologischer Natur, weil Heisenberg eigentlich nur zeigte, dass es intrinsische Begrenzungen dessen, was man messen konnte, gab. Dennoch gaben Heisenberg und fast alle anderen Physiker so Polkinghorne - dem Prinzip eine ontologische Bedeutung. „Es wurde als Prinzip tatsächlicher Unbestimmtheit behandelt, nicht als bloße Unkenntnis.“64

Als Gewährsmann für sein Verständnis des kritischen Realismus verweist Polkinghorne insbesondere auf Michael Polanyi, einen im deutschsprachigen Bereich weniger bekannten Wissenschaftsphilosophen, der selbst aktiver Natur-

58 Aufgrund seiner spärlichen Fußnotensetzung manchmal leider ein grundlegendes Problem bei Polkinghorne.

59 Polkinghorne, One World (1986), 22: „an adequate account of a circumscribed physical domain, a map good enough for some, but not for all, purposes“.

60 Polkinghorne, John C., Reason and Reality, London: SPCK 2011, 23: „... valuable but of recognized inadequacy".

61 Ebd.

62 Polkinghorne, John C., Science and Christian Belief, London: SPCK 1994, 156: „epistemology models ontology“. Vgl. zur Entstehung der Formel Losch, Jenseits der Konflikte (2011), 119 Anm. 85.

63 Polkinghorne, Scientists as Theologians (1996), 14.

64 Polkinghorne, Metaphysics of Divine Action (1995), 147-156, hier 148: „It was treated as a principle of actual indeterminacy, not mere ignorance." Zum möglichen Einfluss Barbours auf diesen Gedankengang vgl. Losch, Jenseits der Konflikte (2011), 120. 
wissenschaftler (Chemiker) war, bevor er sich der Wissenschaftstheorie zuwandte. ${ }^{65}$ Für Polkinghorne stellt bereits dieser (dem eigenen ähnliche) Werdegang eine Auszeichnung seiner Philosophie dar. Formativ für Polkinghornes eigene erkenntnistheoretische Überlegungen ist sodann die Auseinandersetzung mit Andrew Pickering, der die Quantentheorie konstruktivistisch interpretiert. Gegen dessen Behauptung, dass „die Welt der Hochenergiephysik sozial produziert worden ist", ${ }^{66}$ verweist Polkinghorne auf die Reflexionen von W. H. NewtonSmith, der auf die Rolle von subjektiven Urteilen im Forschungsprozess eingeht, und dennoch an einer Wahrheitsähnlichkeit wissenschaftlicher Theorien, also an einer realistischen Orientierung festhält. ${ }^{67}$ Michael Polanyi ist nun derjenige, der diesen Zusammenhang am intensivsten reflektiert hat, und deswegen von Polkinghorne entsprechend wertgeschätzt wird. ${ }^{68}$

\subsection{Parallelen der Bereiche nach Polkinghorne}

Polkinghorne erkennt wie Barbour auf Basis des kritischen Realismus einige Parallelen zwischen Naturwissenschaft und Religion, auch das von Barbour benutzte Whitehead-Zitat findet sich früh. ${ }^{69}$ Sechs Vergleichsmomente kann Polkinghorne zwischen den Bereichen ausmachen.

1.) In beiden Bereichen ist die Bedeutung „wohlwollender Referenz“ hervorzuheben. Die Tatsache, dass „man sich auf dieselbe Realität in unterschiedlichen Sprachen und Modellen beziehen kann“, ${ }^{70}$ bedeutet, dass wir „,Allesoder-nichts'-Theorien über das Wissen widerstehen“ und bereit sein müssen, „auch schrittweise Leistungen zu würdigen“ (JP/104).

2.) Da Wissenschaft von Personen ausgeübt wird und auf den unausgesprochenen Fähigkeiten beruht, die man durch Praxis innerhalb einer Gemeinschaft gewinnt (JP/105), ist der Unterschied zwischen Naturwissenschaft und Theologie nicht grundlegender Art, sondern am jeweiligen Grad der Macht

65 Zu Polanyi vgl. Losch, Jenseits der Konflikte (2011), Kap. 7.

66 Pickering, Andrew, Constructing Quarks, Chicago: University of Chicago Press 1984, 406: „the world of HEP [High Energy Physics] was socially produced“.

67 Newton-Smith, William H., The Rationality of Science, London: Routledge 1981, 183-207, $232-234$.

68 Polkinghorne, John C., Rochester Roundabout. The Story of High Energy Physics, New York: W. H. Freeman 1989, 175.

69 Polkinghorne, One World (1986), 28.

70 Polkinghorne, An Gott glauben (2000), 105. Im Folgenden zitiert durch JP/Seitenangaben in Klammern. 
empirischer Forschung orientiert (JP/112). Bereits Barbours Spektrumsthese hatte sich für eine solche Argumentation ja $u$. a. auf Polanyi bezogen, und eben dies tut Polkinghorne.

3.) Wie es keine bedeutenden naturwissenschaftlichen Fakten gibt, die nicht interpretierte Fakten sind, also eine gewisse Zirkularität im wissenschaftlichen Erkenntnisprozess angenommen werden muss, war der Theologie dieser hermeneutische Zirkel seit langem bekannt; ihr war ,seit langem bewußt, daß man glauben muß, um zu verstehen, ... und daß man verstehen muß, um glauben zu können“ (JP/113). Es war ebenfalls Polanyi, der diese Einsicht der Kirchenväter für die Wissenschaftstheorie fruchtbar gemacht hat.

4.) Die Quantentheorie hat gezeigt, dass es keine universale Epistemologie gibt (JP/107), ${ }^{71}$ Entitäten werden daher auf einem Wege erkannt, der ihrer eigenen spezifischen Natur entspricht. Polkinghorne nennt dies den „epistemischen Zirkel“. Ebenso hat auch Thomas F. Torrance betont, dass Gott nur auf einem Wege erkannt werden kann, der seiner eigenen Natur angemessen ist $(J P / 114)^{72}$ (und das ist der Glaube ${ }^{73}$ ).

5.) In Polkinghornes fünfter Parallele hören wir noch einmal ein Echo der Auseinandersetzungen, die sein Verständnis des kritischen Realismus geformt haben: Wie soziale Faktoren das Wachstum des wissenschaftlichen Wissens beschleunigen oder hemmen können, aber nicht den Charakter dieses Wissens bestimmen (JP/107), ist auch die Theologie nicht einfach eine soziale Konstruktion. Polkinghorne sieht diese Parallele in seinen Überlegungen zu einer revidierten natürlichen Theologie bestätigt (JP/119).

6.) Fundamental ist für Polkinghorne schließlich die Überzeugung, dass die Anschauung des wissenschaftlichen Realismus am besten die (Erfolgs-) Erfahrungen der naturwissenschaftlichen Forschung verstehen lässt. Dies ist sicherlich ein Echo des bekannten Diktums Putnams, nach dem es das positive Argument für den Realismus sei, dass er „die einzige Philosophie ist, die den Erfolg der Wissenschaft nicht zu einem Wunder macht“.${ }^{74}$ Wie gelangt

71 Der Satz vom ausgeschlossenen Dritten gilt nach Werner Heisenberg, der sich hier auf Überlegungen von Carl Friedrich von Weizsäcker bezieht, in der Quantenwelt nicht (Heisenberg, Werner, Physik und Philosophie, Berlin: Ullstein 1959, 152). Damit gibt es eine eigene Quantenlogik, eine eigene Rationalität der Quantenwelt, vgl. Polkinghorne, John C./Welker, Michael (Hg.), The End of the World and the Ends of God, Harrisburg: Trinity Press 2000, 5.

72 Torrance, Thomas F., Theological Science, Oxford: Oxford University Press 1969, 9f. Mehr dazu bei Losch, Jenseits der Konflikte (2011), 144-146.

73 Torrance, Thomas F. (Hg.), Belief in Science and Christian Life. The Relevance of Michael Polanyi's Thought for Christian Faith and Life, Eugene: Wipf and Stock Pub. 1998, 4.

74 Putnam, Hilary, Mathematics, Matter and Method, Cambridge: Cambridge University Press 1975, 73: „the only philosophy which does not make the success of science a miracle“. 
man aber von diesem Punkt aus zu einem theologischen Realismus? Polkinghorne abstrahiert dazu den wissenschaftlichen Realismus dahingehend, dass für diesen Verständlichkeit ein verlässlicher Führer zur Ontologie ist (JP/ 107ff). Diese grundsätzliche Möglichkeit naturwissenschaftlicher Forschung begründet Polkinghorne schließlich mit der Gottebenbildlichkeit des Menschen (JP/119) - dessen Geist den Geist hinter den Strukturen des Universums abbildet, sollte man wohl im Polkinghorne’schen Sinne ergänzen.

Polkinghorne sind nun auch einige Differenzen zwischen dem kritischen Realismus in Naturwissenschaft und Theologie klar vor Augen. Der grundsätzliche Unterschied liegt für ihn - stärker als für Barbour - darin, dass es in den experimentellen Naturwissenschaften immer die Möglichkeit der Wiederholung der Erfahrungen gibt, während die Theologie von Ereignissen und den Einsichten von Personen abhängt, die unwiederholbar und einzigartig sind. ${ }^{75}$ Dies gelte allerdings für alle Formen subjektiver Erfahrung, wie Polkinghorne in einem späteren Werk sagt. ${ }^{76}$ Sie haben 1.) die historische Dimension ernst $\mathrm{zu}$ nehmen. Daneben geht insbesondere die Theologie davon aus, dass 2.) auch der „Gegenstand“ der theologischen Wissenschaft die Initiative ergreifen kann, eine Begegnung mit der Wirklichkeit zu arrangieren (man denke an das Konzept der Offenbarung). Während 3.) die Naturwissenschaft aufgrund der Wiederholbarkeit ihrer Ergebnisse universale Akzeptanz erfährt, ist die religiöse Szene demgegenüber deutlich fragmentierter. 4.) Religiöser Glaube betrifft alle Aspekte des Lebens und befriedigt nicht nur den Intellekt. Glaubensüberzeugungen sind die Ursache von Konflikt und Unterdrückung wie menschlichem Aufblühen und leidenschaftlichem Engagement gleichermaßen. ${ }^{77}$

\subsection{McGraths Verwendung des Konzepts}

Alister E. McGrath ist auf eigenen Wegen zu einer Wertschätzung des kritischen Realismus gelangt, von denen er 2002 in Teil 2 seiner Scientific Theology mit dem Titel Reality Rechenschaft ablegt. ${ }^{78}$ Als ausgebildeter Biochemiker und Theologe kann man ihn sicherlich auch zu den Wissenschaftler-Theologen zählen; dass er von Polkinghorne als solcher nicht aufgeführt wird, verrät wiederum viel über Rezeptionskreise. McGraths Verdacht, die Nichtbeachtung Roy Bhaskars sei ein

75 Polkinghorne, Scientists as Theologians (1996), $18 \mathrm{f}$.

76 Polkinghorne, John C., Quantum Physics and Theology, London: SPCK 2007, 9.

77 Ebd., $10-14$.

78 McGrath, Reality (2002). Im Folgenden zitiert durch MG/Seitenangaben in Klammern. 
Hinweis darauf, „dass wenigstens ein signifikanter Teil der ,Science and Religion Community، sich von den Debatten und Ressourcen der Hauptströmungen in der akademischen Community entfernt hat" (MG/207f.), ${ }^{79}$ ist umgekehrt wiederum ein Hinweis darauf, dass er selbst lange nur am Rande ein Teil dieser „Science and Religion Community“ gewesen ist und hauptsächlich als originärer Theologe gearbeitet hat. Das hat spätestens sein Programm einer Scientific Theology geändert. Er definiert diese wissenschaftliche Theologie gegen Kant als A-posteriori-Disziplin, die darauf antwortet und eine Darstellung dessen bietet, was von Gott durch Offenbarung gewusst werden kann, und dabei die geschichtete Natur dieses Wissens von Gott vollständig anerkennt (MG/xi). Auch er vertritt dabei eine Variante der Spektrumsthese; bereits innerhalb der Naturwissenschaften gebe es ein Spektrum des Zusammenspiels von „Objektivität“ und „sozialer Konstruktion“, wobei diese Konstruktionen hier nicht im postmodernen Sinne zu verstehen, sondern empirisch verankert seien (MG/xiii). All die genannten zentralen Eigenschaften finden sich in Roy Bhaskars kritischem Realismus, auf den McGrath nach eigenen Angaben 1998 gestoßen ist und den er umgehend zu seinem eigenen gemacht hat (MG/xvi).

McGraths Überlegungen setzten eine realistische Perspektive auf die Welt voraus, wie sie die Geschichte der Naturwissenschaften und ihre Erfolge seiner Ansicht nach unterstreichen. „Realismus funktioniert“ („Realism works“) konstatiert McGrath ziemlich pragmatisch, und daher seien die meisten Naturwissenschaftler im Kern Realisten (MG/123). Der wissenschaftliche Realismus sei daher wenigstens teilweise - eine empirische These (MG/124). ${ }^{80}$ Ähnlich wie Barbour grenzt McGrath seinen Realismus sodann vom Idealismus, Positivismus und Instrumentalismus ab und postuliert ergo einen kritischen Realismus (MG/133ff.). Das Attribut „kritisch“ erläutert auch McGrath dabei in Abgrenzung vom naiven Realismus, allerdings ebenso in Abgrenzung vom postmodernen Anti-Realismus (MG/195).

\footnotetext{
Meine Verteidigung des Realismus hat mit der grundlegenden Überzeugung zu tun, dass der Realismus die beste Erklärung der Erfolge und Strategien der Naturwissenschaftler bietet und dass er eindeutig von der klassischen christlichen theologischen Tradition vorausgesetzt und angewandt wird, in der ich stehe (MG/199), ${ }^{81}$
}

79 Orig.: ,that at least a significant section of the ,science and religion' community has become detached from the debates and resources of the mainline academic community".

80 Eine ähnliche Zusammenstellung dieser Gedanken Jarrett Leplins findet man im Übrigen bei Polkinghorne, Rochester Roundabout (1989), 166.

81 Orig.: „my defense of realism has ... to do ... with the basic conviction that realism offers the best explanation of the successes and strategies of the natural sciences and that it is clearly 
ist McGrath überzeugt. Das allerdings beinhalte noch kein Urteil über die Art des Realismus, und hier hält er eben Bhaskars kritischen Realismus für den angemessensten. ${ }^{82}$ Interessant ist es, wie stark McGrath mit Bhaskar betont, dass es weder Methodologie noch Epistemologie erlaubt werden kann, Angelegenheiten der Ontologie zu bestimmen (MG/211f.), vielmehr muss die Methodologie a posteriori von der Ontologie bestimmt werden (MG/218, 223f.). ${ }^{83}$ Bhaskars Realismus umfasst die Naturwissenschaften ebenso wie die Sozialwissenschaften, weil er die Vorstellung einer geschichteten Wirklichkeit vertritt; ${ }^{84}$ da jede Schicht - ob physikalisch, biologisch oder kulturell - als „real“ angesehen werden muss, ist es die Natur des Gegenstandes, welche die Form ihrer möglichen Wissenschaft bestimme (MG/217). ${ }^{85}$

Bhaskars kritischer Realismus wird dann von McGrath - ebenfalls wie bei Barbour - auf die Theologie übertragen:

\begin{abstract}
So wie Naturwissenschaften ein distinktes Vokabular, einen Satz hypothetischer Entitäten und Konstrukte entwickeln, um die Schichten der Wirklichkeit darzustellen, denen sie begegnen, so hat die Christliche Theologie ihre eigene charakteristische Terminologie, Modelle und Konzeptionen hervorgebracht, um die Wirklichkeit darzustellen, welcher in Jesus Christus begegnet wird und welche durch ihn offenbart wird (MG/4). ${ }^{86}$

In einer solchen realistischen Perspektive sehen sowohl die Naturwissenschaften als auch die Christliche Theologie ihre Gegenstände als im Besitz einer Wirklichkeit und intrinsischen Rationalität an, die im Prozess der Beobachtung und Theorieentwicklung erforscht und anerkannt werden (MG/4). ${ }^{87}$
\end{abstract}

presupposed and applied by the classic Christian theological tradition, within which I stand." Der Nominalismus kommt dabei in dem ganzen Buch McGraths merkwürdigerweise nicht vor.

82 Vgl. seine Darstellung der Philosophie Bhaskars, ebd., $209 \mathrm{ff}$.

83 Dies hat eine antireduktionistische Zielrichtung, wird jedoch nicht mit der Formel Polkinghornes (,epistemology models ontology“), der sonst positiv rezipiert wird (ebd., 206f.), in Beziehung gesetzt.

84 Herkunft dieser Vorstellung ist möglicherweise die Philosophie Polanyis, vgl. Losch, Jenseits der Konflikte (2011), 130 Anm. 141.

85 Mit Zitat aus Bhaskar, Roy, The Possibility of Naturalism, Brighton: Harvester Press 1979, hier 3: "It is the nature of the object that determines the form of its possible science.“

86 Orig.: „Just as the natural sciences develop a distinct vocabulary, set of hypothetical entities and constructs to represent the levels of reality they encounter, so Christian theology has evolved its own characteristic terminology, models and conceptualities to represent the reality which is encountered and disclosed in Jesus Christ.“

87 Orig.: „On such a realist view, both the natural sciences and Christian theology regard their objects as possessing a reality and intrinsic rationality which are to be ascertained and respected in the process of observation and the development of theories." 
Auch die Theologie vertrete mit ihrer Unterscheidung der Wirklichkeit Gottes (Essenz) von der kontingenten Wirklichkeit der Schöpfung (Existenz) die Konzeption einer geschichteten Wirklichkeit, deren Ontologie die auf sie passende Methodologie bestimme, und deswegen sei der kritische Realismus auch auf diese anwendbar (MG/228).

Der Realismus, der von den Naturwissenschaften verlangt wird, wird daher entsprechend für eine wissenschaftliche Theologie verlangt, insofern als beide danach suchen, eine Ratio oder einen Logos aufzudecken, der ihren Gegenständen eigentümlich ist und der vor und unabhängig von ihren Beobachtungen existiert (MG/267f.). ${ }^{88}$

Vollständig werde der Logos erst in Christus enthüllt, wie McGrath auf einer Linie mit Barth und Torrance betont (MG/267).

McGraths weiterführende Idee der Gegenstandsadäquatheit ist im Grunde einer der von Polkinghorne genannten Parallelen zwischen den Bereichen nicht unähnlich. Tatsächlich handelt es sich dabei um die Rezeption eines über Torrance vermittelten Grundgedankens von Karl Barth; ${ }^{89}$ der Gedanke ist als ein Erbe der hermeneutischen Tradition ebenso auch bei Roy Bhaskar zu finden. ${ }^{90}$

\section{Anfragen an den theologischen Realismus}

Der Philosoph Ernan McMullin hat die Übertragbarkeit eines wissenschaftlichen kritischen Realismus auf die Theologie aus verschiedenen Gründen abgelehnt. ${ }^{91}$ In einem Vergleich von Peacockes Argumenten für einen wissenschaftlichen Realismus mit denen für einen theologischen Realismus kommt er $\mathrm{zu}$ dem Schluss, dass „die Schwierigkeiten auf dem Wege jedweder Sorte von Realismus in der Theologie beachtlich sind“. ${ }^{92}$ 1.) Bereits die Annahme einer geschichteten Wirklichkeit, in der Naturwissenschaft und Theologie nur verschiedene Ebenen darstellen, lehnt er ab; er zieht es vor, von verschiedenen Bereichen der Wirklichkeit zu sprechen (MM/39). 2.) Nur die natürliche Theologie macht für ihn den

88 Orig.: „The realism that is mandated by the natural sciences is thus correspondingly mandated for a scientific theology, in that both seek to uncover a ratio or logos which is peculiar to their object, and which exists prior to and independent of their observations."

89 Vgl. Losch, Jenseits der Konflikte (2011), 250.

90 Bhaskar, Possibility of Naturalism (1979), 23.

91 Vgl. zum Folgenden Losch, Jenseits der Konflikte (2011), $158 \mathrm{ff}$.

92 McMullin, Ernan, „Realism in Theology and Science. A Response to Peacocke“. In: Religion \& Intellectual Life 2/4 (1985), 39-47. Im Folgenden zitiert durch MM/Seitenangaben in Klammern. Hier 45: „the difficulties in the way of any sort of realism in theology are thus formidable“. 
Eindruck, intersubjektiv zu sein und 3.) kein Typ der Theologie schreitet stetig voran (MM/42f.). Ein zentrales Argument für den Realismus in der Naturwissenschaft ist 4.) ihre Erfolgsgeschichte, die erklärt werden muss, aber es gibt kein entsprechendes Argument für irgendeinen Typ der Theologie (MM/43). Der einzige Grund, eine Affinität zwischen Naturwissenschaft und Theologie zu betonen, den er anerkennt, ist 5.) der generelle metaphorische Charakter der zentralen explanatorischen Konzepte in beiden (MM/45). Doch „der Grund, warum die Sprache der Theologie für analog gehalten wird (,metaphorisch' im erweiterten zeitgenössischen Sinne) liegt nicht in ihren Abläufen, sondern im Charakter ihres Gegenstands begründet“ (MM/46). Mehr noch ist Vieles der Sprache in der Naturwissenschaft keinesfalls metaphorisch. ${ }^{93} 6$.) Schlussendlich unterstützt McMullin Peacockes These, dass die Gegenstände der Naturwissenschaft jenseits der Reichweite wörtlicher Beschreibung liegen, wie es die Gegenstände der Religion auch tun. Dennoch hält er es nicht für klug, die Parallelen weiter auszuziehen (MM/47). Daraus ist zu schließen, dass eine direkte Linie zwischen wissenschaftlichem und theologischem Realismus hochproblematisch ist.

Dies ist auch die Schlussfolgerung von Willem Drees. In seiner Kritik von Peacocke deutet er auf dessen Schluss a minore ad majus, in dem Peacocke auf das starke Programm in der Wissenssoziologie verweist und argumentiert:

Diejenigen, die diese Ansicht vertreten, würden $a$ forteriori eine ähnliche Perspektive auf die kognitiven Behauptungen der Theologie einnehmen. Der Versuch, Wissenschaft und Theologie aufeinander $\mathrm{zu}$ beziehen, würde dann auf die Herstellung eines Zusammenhangs zwischen zwei Ideologien reduziert und würde auf diese Weise selbst zu einer rein soziologischen Untersuchung oder Beschäftigung mit der Ideengeschichte. ${ }^{94}$

Drees begreift die Furcht, die sich hier äußert, wie folgt:

Falls schon die Naturwissenschaft kein Wissen über die Wirklichkeit darstellen würde, wie viel mehr müssten wir dies im Falle der Theologie fürchten, wo die symbolische Natur der Sprache um einiges beharrlicher ist und die Interaktion mit der Wirklichkeit, die erhofft wird, weit weniger greifbar. ${ }^{95}$

93 Wir haben gesehen, dass bereits Polkinghorne kritisch gegenüber einem zu metaphorischen Ansatz der Naturwissenschaft war.

94 Peacocke, Arthur, Theology for a Scientific Age. Being and Becoming - Natural and Divine, Oxford: Blackwell 1990, 19: „Those having this view would adopt a forteriori a similar view of the cognitive claims of theology and then the exercise of relating science and theology would be reduced to that of relating two ideologies and so would itself become a purely sociological inquiry or exercise in the history of ideas."

95 Drees, Willem B., Religion, Science and Naturalism, Cambridge: Cambridge University Press 1996, 145: „If science were not knowledge of reality, how much more would we have to fear that this 


\section{Von hier aus folgert er,}

dass man nicht die problematische Verteidigung des theologischen Realismus vermeiden kann, indem man sich der einfacheren, aber für das beabsichtigte Ziel irrelevanten Verteidigung des wissenschaftlichen Realismus zuwendet. Welche Perspektive auf den mit ihrem Erfolg und ihrer andauernden Offenheit für Entwicklung und Wandel komplexen Charakter der Naturwissenschaft auch siegen wird, der theologische Realismus muss immer noch aus sich selbst heraus gerechtfertigt werden. ${ }^{96}$

Dies ist der Grund, warum Drees’ Reaktion auf McMullins Kritik bestätigend ist:

Angesichts der Unterschiede im Erfolg können wir den theologischen Realismus nicht auf dieselbe Weise wie den wissenschaftlichen Realismus verteidigen. Deshalb können wir das Prestige der Naturwissenschaft nicht auf diese Weise für die Theologie kooptieren. ${ }^{97}$

\subsection{Van Huyssteens Replik}

J. Wentzel van Huyssteen scheint dies anders zu sehen und definiert dazu zentrale Begriffe, die von McMullin kritisiert worden waren, auf kreative Weise neu. Seine Ideen stellen eine direkte Antwort auf McMullins Entgegnungen dar und sind auch als weitere Unterstützung für den Kritischen Realismus zitiert worden (JP/120).Van Huyssteens Interpretation ist inspiriert von der Überzeugung, dass „der wissenschaftliche Realismus, in der Form eines qualifizierten kritischen Realismus, hervorragende Ressourcen besitzt, um die Verlässlichkeit und Gültigkeit von theologischen Annahmen zu unterstützen“.98 Da die Überzeugung, dass das, wovon wir uns provisorisch in der Theologie eine Vorstellung machen, wirklich existiert, den Grund darstellt, den Begriff des „kritischen Realismus“ zu benutzen

is the case in theology, where the symbolic nature of language is much more persistent and the interaction with the reality hoped for is far less tangible."

96 Ebd., 146: „One cannot avoid the problematic defence of theological realism by turning towards the easier, but for the purpose at hand irrelevant, defence of scientific realism. Whichever view on the complex character of science with its success and its persistent openness to development and change is to prevail, theological realism would still have to be justified on its own terms.“

97 Ebd., 142: „Given the differences in success, we cannot defend theological realism in the same way that scientific realism is defended. Hence, we cannot co-opt the prestige of science for theology in this way."

98 Huyssteen, J. Wentzel van, Theology and the Justification of Faith, Grand Rapids: Eerdmans 1989. Im Folgenden zitiert durch WH/Seitenangaben in Klammern. Hier 155: „scientific realism, in the form of a qualified critical realism, has tremendous resources for supporting the reliability and validity of theological assertions". 
(WH/155), erlaube sie Theologen wie Naturwissenschaftlern anzunehmen, dass sie auf eine gültige, progressive und daher erfolgreiche Weise theoretisieren (WH/155). Er definiert also das Erfolgsargument hinsichtlich der Theologie neu, indem er es mit einer revidierten Auffassung von Fortschritt verbindet, insofern

manche Theorien in der Theologie mit guten Gründen behaupten können, bessere Theorien als andere konkurrierende Theorien zu sein, weil sie entsprechend dem spezifischen epistemologischen Status des biblischen Texts, wie auch entsprechend der Natur des Gelesenen und der Interpretation, die dieser Text fordert, bessere Erklärungen bereitstellen (WH/161). ${ }^{99}$

Kurz gesagt: „Theologischer Fortschritt bedeutet erneuertes aber auch besseres Verstehen der biblischen Nachricht in jedem neuen Kontext und jeder neuen Problemsituation“ (WH/194). ${ }^{100}$ Einen ähnlichen Weg geht er in der Frage der Wirklichkeitsabbildung:

... die Tatsache, dass Theologie nach und nach mehr über Gott herausbekommen kann, indem sie epistemologisch immer gültigere Aussagen über ihn macht, habe ich als Wirklichkeitsabbildung in einem kognitiven Sinne zu definieren versucht (WH/162). ${ }^{101}$

Van Huyssteen akzeptiert McMullins Einwand, dass Naturwissenschaft und Theologie eher verschiedene Bereiche denn verschiedene Ebenen behandeln (WH/ 156), und er unterstützt auch McMullins Idee, dass es ein Gebiet gibt, auf dem sich die beiden überlappen, welches auf der Ebene der Reflexion menschlichen Wissens ist. Hier „hat jeder etwas wichtiges zu sagen über zwei sehr verschiedene, aber auch sehr bedeutsame Bereiche der Wirklichkeit“ (WH/156). ${ }^{102}$ Van Huyssteen glaubt, dass es auf dieser Ebene des Problems der Verlässlichkeit theologischen Wissens ist, dass eine Theorie des kritischen Realismus getestet werden müsse, und dass McMullin in diesem Sinne auch mit der Behauptung sympathisieren würde, „dass sowohl Naturwissenschaft als auch Theologie als ,realistisch` betrachtet werden können, also dass sie verlässliche Wahrheitsbehaup-

99 Orig.: ,in terms of the fact that some theories in theology can with good reasons claim to be better theories than other competing theories, since they provide better explanations, because of the specific epistemological status of the biblical text, as well as the nature of the reading and interpretation this text requires.“

100 Orig.: „Theological progress means renewed but also better understanding of the biblical message in each new context or problem situation."

101 Orig.: „the fact that theology can gradually get to know more about God by making more epistemologically valid statements about Him, is what I have tried to define as reality depiction in a cognitive sense."

102 Orig.: „each has something important to say about two very different but also very important domains of reality“. 
tungen über Bereiche der Wirklichkeit machen, die jenseits unserer Erfahrung liegen“ (WH/156). ${ }^{103}$

McMullin jedoch sagte, dass er die These unterstützen würde, dass die Gegenstände der Naturwissenschaft wie der Religion jenseits der Reichweite wörtlicher Beschreibung liegen. Offensichtlich ist van Huyssteens Rettungsversuch des theologischen kritischen Realismus etwas bemüht, und er ist auch später ein Stück weit vom kritischen Realismus abgerückt. ${ }^{104}$

\subsection{Kritik des kritischen Realismus}

McMullins und Drees' Kritik richtete sich auf Peacockes Verständnis des kritischen Realismus, aber auch die ähnlichen Ansätze Barbours und Polkinghornes sind ebenso wie McGraths Neuansatz auf Basis Bhaskars davon betroffen, wenn das Schichtenmodell der Wirklichkeit dahingehend in Frage gestellt wird, ob die theologische Wirklichkeit hier wirklich als eigene Ebene oben aufgesattelt oder als untere Basis postuliert (McGrath) werden kann. ${ }^{105}$ Sicher haben die Naturwissenschaften vielfach Hierarchien in der Natur beobachtet, doch ob sich die Theologie und die Geisteswissenschaften so einfach als weitere Ebenen diesen Hierarchien zuordnen lassen, ist doch eine eher weltanschauliche Frage. ${ }^{106}$

Man könnte außerdem die Kritik McMullins dahingehend noch weiter schärfen, dass auch eine natürliche Theologie keine intersubjektive Geltung beanspruchen kann, weil sie z. B. in der von Karl Barth geprägten protestantischen Theologie recht grundsätzlich abgelehnt wird. Schließlich bleibt die Frage, wie theologischer Fortschritt bzw. Erfolg aussehen soll, oder ob zumindest im theo-

103 Kursiv vom Verfasser. Orig.: „that both science and theology could be regarded as ,realist“, that is, as making reliable truth claims about domains of reality that lie beyond our experience“. 104 Huyssteen, J. Wentzel van, The Shaping of Rationality. Toward Interdisciplinarity in Theology and Science, Grand Rapids: Eerdmans 1999, 213-216.

105 Grundsätzlich scheint McGrath die Kritik McMullins am theologischen kritischen Realismus auch nicht wahrgenommen zu haben, denn er zitiert den zentralen kritischen Aufsatz McMullins in Reality (2002) nicht.

106 Vgl. z. B. die alternative Darstellung von Dinter, Astrid, Vom Glauben eines Physikers, Mainz: Matthias-Grünewald-Verlag 1999, 61 ff., und die Kritik von Link, Christian, „Die Wahrnehmung der Natur als Schöpfung. Zum Modell eines Dialogs zwischen Naturwissenschaft und Theologie im Anschluss an Viktor von Weizsäcker“. In: Zufall Mensch? Das Bild des Menschen im Spannungsfeld von Evolution und Schöpfung, hrsg. v. L. Klinnert, Darmstadt: WBG 2007, 62f. 
logischen Bereich nicht eine kontextuelle Kohärenztheorie angemessener wäre. ${ }^{107}$ Niels Henrik Gregersen formuliert das so:

... die Rolle der Theologie ist nicht an erster Stelle, Daten im Wettbewerb mit den Naturwissenschaften zu ,erklären', sondern eine Welt wieder zu beschreiben, die bereits von den Naturwissenschaften beschrieben und (teilweise) erklärt worden ist. ${ }^{108}$

Er unterstützt mit seinem Ansatz einen metaphysischen und semantischen Realismus, lehnt aber in Bezug auf Theologie und Geisteswissenschaften einen theoretisch-explanatorischen Realismus, wie er der Vorstellung einer Konvergenz der Wahrheitsähnlichkeit zugrunde liegt, ab. ${ }^{109}$ Er weist außerdem darauf hin, dass alle Analogien zwischen einem common sense Realismus und theologischen Realismus dort zusammenbrechen, wo man bedenkt, dass Gott nicht nur das Objekt, sondern auch das Subjekt menschlichen Denkens darstellt. ${ }^{110}$ Auch ein überzeugter kritischer Realist wie Kees van Kooten Niekerk kann die Legitimation eines theologischen Realismus nur im Kontext des Glaubens anerkennen. ${ }^{111}$ Peacockes Idee des Kritischen Realismus als Mittelweg zwischen naivem Realismus und dem starken Programm in der Wissenssoziologie wird schließlich von der Religionsphilosophin Nancey Murphy dahingehend kritisiert, dass diese Konzepte verschiedenen Dimensionen in einem „konzeptionellen Raum“ zugehörig und daher nicht in Beziehung zu setzen seien. ${ }^{112}$

Christian Link lehnt den kritischen Realismus aus einem formalen ${ }^{113}$ und einem inhaltlichen Grund ab. Er bezieht sich auf dessen Polkinghorn'sche Aus-

107 Vgl. Gregersen, Niels Henrik, „A Contextual Coherence Theory for the Science-Theology Dialogue“. In: Rethinking Theology and Science. Six Models for the Current Dialogue, hrsg. v. dems./J. W. van Huyssteen, Grand Rapids: Eerdmans 1998, 181-231.

108 Gregersen, Niels Henrik, „Critical Realism and Other Realisms“. In: Fifty Years in Science and Religion. Ian G. Barbour and His Legacy, hrsg. v. R. J. Russell, Aldershot Hants: Ashgate 2004, 77-96, Zitat 78: „... the role of theology is not primarily to ,explain' the data in competition with the sciences, but to redescribe a world already described and (partially) explained by the sciences." 109 Für eine Definition dieser drei Realismen, die der wissenschaftliche Realismus beinhaltet, siehe Psillos, Stathis, Scientific Realism. How Science Tracks Truth, London: Routledge 1999, xix. 110 Gregersen, Critical Realism (2004), 83.

111 van Kooten Niekerk, Kees, „Critical Realism“. In: Encyclopedia of science and religion, Bd. I, hrsg. v. J. W. van Huyssteen, New York: Macmillan 2003, 190-193.

112 Murphy, Nancey, Anglo-American Postmodernity, Boulder: Westview Press 1997, 45.

113 Naturwissenschaftliche Erfahrung sei wiederholbar und verfügbar, die religiöse Gotteserfahrung grundsätzlich nicht. Allerdings erkennt wie dargestellt auch Polkinghorne diese Differenz der Einzigartigkeit religiöser Erfahrung an, und dies hindert ihn nicht daran, am Kritischen Realismus festzuhalten. 
prägung und argumentiert, er müsse sich an seinem eigenen Anspruch messen lassen,

„ob (und wie) sich eine Übereinstimmung oder wenigstens eine Korrespondenz von Epistemologie und Ontologie aufweisen lässt, d. h. ob sich klären lässt, inwiefern sich naturwissenschaftliche und theologische Aussagen auf denselben Wirklichkeitszusammenhang beziehen“. ${ }^{114}$

Im Anschluss an Hans-Dieter Mutschler benennt er dafür den inhaltlichen Grund, dass den physikalischen Gleichungen kein Hinweis auf ein Telos der Welt zu entnehmen sei, ohne ein teleologisches Argument aber kein Brückenschlag von der Physik her zur Theologie zu bewältigen sei. ${ }^{115}$ Grundsätzlich wäre also eine naturphilosophische Finalität eine Brücke, die bei einer gewissenhaft verstandenen Naturwissenschaft zwar von der Theologie ausgeht, aber kein anderes Ufer findet. Allerdings hält der von Link angeführte Mutschler die Vermittlung zwischen Physik und Theologie durch eine Metaphysik grundsätzlich für möglich. ${ }^{116}$

Wenn Wolfgang Schoberth darin zuzustimmen ist, dass „die Alternative von Realismus und Nominalismus oder Realismus und Idealismus aufgelöst werden“ muss, ${ }^{117}$ erscheint dies umso notwendiger, wenn im deutschsprachigen Bereich genauso engagiert ein Nominalismus oder Konstruktivismus denn Realismus im Gespräch von Theologie und Naturwissenschaften propagiert werden kann. ${ }^{118}$ In Auseinandersetzung mit Jürgen Hübners „kritischem Nominalismus“119 habe ich ansatzweise versucht, die Forderung Schoberths mit der Idee eines „konstruktivkritischen Realismus“ umzusetzen. ${ }^{120}$ Drei Dinge scheinen mir hier insbesondere

114 Link, Wahrnehmung der Natur als Schöpfung (2007), 62.

115 Ebd., 63, mit Verweis auf Mutschler, Hans-Dieter, Physik und Religion. Perspektiven und Grenzen eines Dialogs, Darmstadt: WBG 2005, $259 \mathrm{f}$.

116 Ebd., 262.

117 Schoberth, Wolfgang, „Das Universum und die Welt, in der wir leben. Systematisch-theologische Überlegungen zur Kosmologie“. In: Theologie und Kosmologie. Geschichte und Erwartungen für das gegenwärtige Gespräch, hrsg. v. J. Hübner/I.-O. Stamatescu/D. Weber, Tübingen: Mohr Siebeck 2004, 333-353, Zitat 350.

118 Link, Wahrnehmung der Natur als Schöpfung (2007); Hübner, Jürgen, Theologie und biologische Entwicklungslehre. Ein Beitrag zum Gespräch zwischen Theologie und Naturwissenschaft, München: C. H. Beck 1966; Audretsch, Welt der Quanten (2008); Körtner, Schöpfung und Evolution (2010), 26.

119 Losch, Jenseits der Konflikte (2011), 102-104.

120 Ebd., 157 f., 242 ff. Dazu jetzt auch Link, Christian, Schöpfung. Exegese - Theologie - Kontroversen: Ein theologischer Entwurf im Gegenüber von Naturwissenschaft und Ökologie, Neukirchen-Vluyn: Neukirchener Verlag 2012, 166-168. 
bezogen auf die Barbour'sche Rezeptionsschiene des kritischen Realismus von Bedeutung:

1.) Der kritische Realismus steht im Grunde nur für die postulierten Parallelen zwischen Naturwissenschaft und Theologie. Unterschiede wie überhaupt geisteswissenschaftliche Eigenheiten werden zwar teilweise genannt, aber nach Möglichkeit eingeebnet. Dafür ist Barbours Spektrumsthese beredtes Beispiel. Dagegen ist die eigenständige Qualität des Geisteswissenschaftlichen wieder stark zu machen. McGraths an Bhaskar orientierter Ansatz erscheint aufgrund seiner konsequenten Orientierung an der Natur des jeweiligen Gegenstands zwar angemessener, doch auch hier bleiben Fragen offen.

2.) Der kritische Realismus ist zu Recht dahingehend kritisiert worden, dass er letztlich am cartesischen Zweifel festhält. ${ }^{121}$ Wenn man aber seine Grundidee eines Bewusstseins unserer selbst, „das aus einer Übereinstimmung, Verbindung und Teilhabe an Prozessen entsteht, die uns übersteigen“122 verwirklichen will, müsste man konsequenter an der epistemologischen Aufhebung der Subjekt/Objekt-Schranke arbeiten.

3.) Vielleicht könnte die von Mutschler als Desiderat geforderte Vermittlung von Kausalität und Finalität ansatzweise dort gelingen, wo man eben diese Schranke aufhebt und dem Konzept des kritischen Realismus ein finales Element hinzufügt. Bei einer konsequenten Verschränkung von Subjekt und Objekt erscheint mir dies naturgemäß durch die Bedeutung der menschlichen Intentionalität als Teil der Natur gegeben. ${ }^{123}$

Der Kritische Realismus ist viele Jahre das dominante Paradigma des angelsächsischen Dialogs zwischen Theologie und Naturwissenschaften gewesen. Die Frage ist berechtigt, ob er - wenn auch er auf diese grundsätzliche Ebene immerhin vorstößt - die cartesische Spaltung gegen seine eigene Intention nicht ungewollt perpetuiert.

121 Robbins, J. Wesley, „Pragmatism, Critical Realism and the Cognitive Value of Religion and Science“. In: Zygon 34/4 (1999), 655-666.

122 Vgl. Anm. 38.

$123 \mathrm{Zu}$ berücksichtigen sind hier die Gedanken von Weizsäcker, Victor von, Der Gestaltkreis. Theorie der Einheit von Wahrnehmen und Bewegen, Stuttgart: Thieme ${ }^{6} 1996$; vgl. dazu Losch, Jenseits der Konflikte (2011), Kap. 9, bes. $223 \mathrm{ff}$. 